\title{
Epidemiological insights into the public health burden of sleep disordered breathing: sex differences in survival among sleep clinic patients
}

\author{
Terry Young, Laurel Finn
}

Recognition that sleep disordered breathing (SDB) is prevalent but largely undiagnosed in adults has prompted the needs to assess the public health burden of this disorder and devise appropriate prevention and intervention strategies. ${ }^{12}$ Before this can be accomplished, both population and clinic-based epidemiology studies are required to understand the natural history, risk factors and adverse health sequelae of this disorder, and to determine what severity levels are of clinical and public health significance. In this report, epidemiological advances and research needs are briefly summarised. In the second part of this paper we focus on the need for epidemiological research into sex differences in the natural history of SDB and present new findings that suggest women with SDB may have poorer survival than men.

\section{Occurrence}

Although prevalence estimates of SDB vary according to definitions and methods of measurement, there is general agreement among the most rigorous studies that the prevalence of SDB meeting clinical criteria for sleep apnoea syndrome is in the range of $2-4 \%{ }^{3}$ In the largest population based study conducted with in-laboratory polysomnography, the prevalence of SDB in middle aged adults in the USA based on lesser severity-for example, an apnoea/hypopnoea index (AHI) of 5 or more-has been estimated at $9 \%$ for women and $24 \%$ for men. ${ }^{4}$ Other studies conducted elsewhere in the western world have yielded similar estimates. ${ }^{5}$ In addition to establishing the high prevalence of SDB, these population-based studies have shown, as is evident in the preceding prevalence estimate, that this is not a disease predominantly of men. Furthermore, women with SDB have been shown to have the same symptoms, risk factors, and health correlates as men. ${ }^{6}$ The discrepancy between the ratio of men to women with SDB in clinic patient populations $(8: 1)$ and in the general population $(2-3: 1)$ has underscored the need for more research on women as well as investigations into health care barriers experienced by women with SDB. Also noteworthy is the occurrence of a wide severity spectrum of unrecognised SDB in the population as this indicates that at present even severe cases of SDB are being missed. ${ }^{4}$

\section{Risk factors}

Epidemiological studies to identify risk factors are needed to target people who are most vulnerable to SDB as well as to provide aetiologi- cal clues. Thus far, investigations of obesity as a risk factor have shown that increasing values on most measures of body habitus-including body mass index, waist, hip and neck circumferences, circumference ratios, and skinfold thickness-are strongly related to $\mathrm{SDB}^{34}$ Some studies suggest that body fat distribution in the neck or centrally is stronger than obesity per se as a risk factor for $\mathrm{SDB}^{5}$; further research on this should be valuable in understanding the mechanism by which body habitus influences SDB. Most relevant to case finding or screening, however, is that morbid obesity only accounts for a fraction of the population prevalence of SDB. Moderate or even mild obesity, a common condition in the population, is a significant predictor of SDB. Other hypothesised SDB risk factors which have received some support from population or clinic-based studies include ageing, craniomandibular deviations, smoking, alcohol, familial predisposition, chronic rhinitis, and menopause. ${ }^{35}$

\section{Outcomes}

Epidemiological studies of outcomes are particularly important in assessing the cost of untreated SDB to society, as well as to the sufferer. Although findings are far from conclusive, population-based and clinic-based studies have linked SDB determined objectively (for example, by polysomnography) or subjectively (for example, reported snoring) with behavioural and cardiovascular morbidity. There is no controversy on excessive daytime sleepiness as an outcome of severe SDB. However, there is little information on the association of mild or asymptomatic SDB and hypersomnolence, or on associations of SDB with cognitive impairment. Limited data support a role of SDB in motor vehicle accidents. ${ }^{35}$

Most outcome studies have been focused on investigating associations of SDB and cardiovascular morbidity and mortality. Some, but not all, population-based and clinic-based studies have provided support for a link between snoring or objectively measured SDB and myocardial infarction, angina, coronary heart disease, and stroke. ${ }^{7}$

Several studies of SDB and blood pressure have been conducted. Many clinic-based studies have found associations between SDB and hypertension that become non-significant after controlling for confounding factors such as obesity. Both positive and negative findings have resulted from population-based studies. ${ }^{7}$

A primary goal of the Wisconsin Sleep Cohort Study, an ongoing prospective study of the natural history of SDB, has been to
Correspondence to: Dr T Young. 
Table 1 Mean (SE) blood pressure by apnoea/hypopnoea index (AHI) category in participants in the Wisconsin Sleep Cohort Study

\begin{tabular}{|c|c|c|c|c|}
\hline \multirow[b]{2}{*}{ Blood pressure ( $\mathrm{mm} \mathrm{Hg}$ ) } & \multicolumn{4}{|l|}{$A H I$} \\
\hline & $<5$, not habitual snorer & $<5$, habitual snorer & $5-15$ & $>15$ \\
\hline \multicolumn{5}{|c|}{ Standard, in-laboratory $(n=708)$} \\
\hline \multicolumn{5}{|c|}{ Early evening } \\
\hline Systolic & $121.9(0.7)$ & $125.6(0.8)^{\star \star}$ & $124.8(1.3)^{\star \star}$ & $127.1(1.7)^{\star \star}$ \\
\hline Diastolic & $80.1(0.5)$ & $82.0(0.6)^{\star \star}$ & $82.2(0.9)^{\star \star}$ & $82.9(1.2)^{\star \star}$ \\
\hline \multicolumn{5}{|l|}{ Morning } \\
\hline Systolic & $119.8(0.8)$ & $122.9(0.9)^{\star \star}$ & $123.4(1.4)^{\star \star}$ & $125.2(1.9)^{\star \star}$ \\
\hline Diastolic & $81.4(0.6)$ & $82.1(0.6)$ & $83.5(0.9)$ & $83.3(1.3)$ \\
\hline \multicolumn{5}{|l|}{ Ambulatory $(n=551)$} \\
\hline \multicolumn{5}{|l|}{ Average during wake } \\
\hline Systolic & $122.7(0.7)$ & $124.0(0.9)$ & $127.5(1.2)^{\star \star}$ & $127.6(1.8)^{\star \star}$ \\
\hline Diastolic & $75.5(0.5)$ & $75.4(0.6)$ & $77.8(0.8)^{\star \star}$ & $78.2(1.0)^{\star \star}$ \\
\hline \multicolumn{5}{|l|}{ Average during sleep } \\
\hline Systolic & $104.2(0.7)$ & $104.8(0.9)$ & $108.2(1.2)^{\star \star}$ & $108.9(1.9)^{\star \star}$ \\
\hline Diastolic & $61.1(0.8)$ & $60.3(0.6)$ & $62.7(0.8)^{\star \star}$ & $62.8(1.3)^{\star \star}$ \\
\hline
\end{tabular}

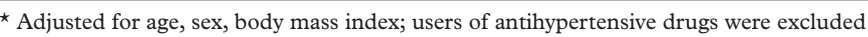

$\star \star \mathrm{p}<0.05$ for difference in means compared with AHI $<5$.

determine, with ample study power and precise measures, the independent association of SDB and blood pressure. Using a two stage random sampling method, a cohort of employed adults was recruited for in-laboratory polysomnography and other tests at four year intervals. ${ }^{4}$ Blood pressure has been determined at different times using different measurement methods. Data summarised from two previous reports $^{89}$ on mean blood pressure from inlaboratory standard sphygmomanometry and ambulatory monitoring by SDB severity category are shown in table 1 . The mean pressures, based only on those not on antihypertensive medication, are adjusted for sex, age, and body mass index . Numerous other variables, including smoking and alcohol use, were investigated for confounding, but none affected the association of SDB and blood pressure. We explored several indicators of body habitus, including neck circumference, waist:hip ratio, and skin fold thicknesses to control confounding due to obesity. All these variables, entered singly and in combination in the regression models, reduced the association of SDB and blood pressure as expected. These variables are highly intercorrelated and, as control variables in the regression models, produced nearly identical results. We selected BMI for the final model because of its more widespread use in predicting cardiovascular outcomes. The consistent trends for increasing blood pressure with increasing SDB severity support a significant but modest association of SDB and blood pressure, independent of age, sex, and obesity.

Without ample study power, an appropriate sample, and proper modelling, small associations are extremely difficult to detect. It is highly likely that inadequate study power and other limitations account for previously reported negative or insignificant associations between SDB and raised blood pressure. Thus, in spite of lack of agreement of previous studies with our findings, we conclude that a true positive association, independent of age, sex, and body mass index or other measures of obesity, does indeed exist.

\section{Gaps}

There are several important epidemiological aspects of SDB that need attention. An obvious gap in current knowledge is an understanding of whether there is a causal role of SDB in the development or exacerbation of health outcomes. The effect of ageing on SDB remains a puzzle; several authors have speculated on whether SDB is the same condition in both middle-aged and older populations. Little is known about SDB in children. Information on the costs and benefits of intervention in the natural history of SDB will be extremely important in addressing future screening and treatment strategies. For this, studies of the effects of risk factor reduction on SDB severity and on measuring the impact of SDB treatment on patient centred outcomes as well as clinical outcomes will be needed. Clinic-based epidemiology studies are needed to understand how the health care system is currently responding to patients seeking help for or with SDB, and to determine the consequences of current case finding, referral, evaluation and treatment. Such information will be a basis for determining what changes are needed to respond to the large number of people with SDB who are not receiving care. Special emphasis must be placed on investigating sex differences in the provision of heath care for this disorder.

As noted above, the lower than expected ratio of men to women with SDB in the general population has raised concerns that there is a selection bias for referral or evaluation that favours men. Sex biases in health care have been noted for several chronic diseases. Studies of cardiovascular disease, AIDS, and end stage renal disease have suggested that women have more severe disease or disease of longer duration than men before they receive attention and that they are not as aggressively evaluated and treated. ${ }^{10}$ To date, there has been no information on whether there is a sex difference in the severity of newly diagnosed SDB, in subsequent treatment, or in clinical outcomes. To begin to look at the current status of medical care for women with sleep apnoea, we ascertained the survival of men and women five 
Table 2 Sex-specific proportions of patients diagnosed with sleep apnoea syndrome by apnoea/hypopnoea index (AHI) in sleep clinic sample $(n=354)$

\begin{tabular}{lcc}
\hline \multirow{2}{*}{ AHI category } & \multicolumn{2}{c}{$\%$ Diagnosed with sleep apnoea syndrome } \\
\cline { 2 - 3 } & Men & Women \\
\hline$<5$ & 4 & 5 \\
$5-20$ & 76 & 40 \\
$>20$ & 99 & 100 \\
\hline
\end{tabular}

years after determination of their SDB status. A brief report of the study method and our findings follow.

\section{Mortality follow up study of men and women with newly diagnosed SDB}

All patients, at least 21 years of age, evaluated at the University of Wisconsin Sleep Disorders Clinic for a sleep disorder by overnight polysomnography from June 1986 to December 1989 who were not previously treated for SDB were identified. The total sample of 354 patients included 91 women $(25.7 \%)$. Of the total sample, $68 \%$ of the men and $44 \%$ of the women were given a diagnosis of sleep apnoea syndrome (SAS). Of the patients that were given a diagnosis of SAS, the mean AHI was 58.2 for men and 50.7 for women (not significantly different). The mean age of patients with SAS was 50.4 years for men and 51.1 years for women compared with 44.8 years for men and 46.7 years for women who were not diagnosed as having SAS. Thus, the mean ages did not differ by sex, and for both men and women, those with a diagnosis of SAS were older than those without. Nearly all men and women with an AHI of $>20$ were diagnosed with SAS, but a lower proportion of women than men with an AHI in the range of 5-20 were given a diagnosis of sleep apnoea syndrome (table 2).

Vital status as of March 1994 was ascertained by searching state mortality records from January 1987 to March 1994. All deaths occurring in Wisconsin, and out of state deaths of people who were Wisconsin residents at the time of their death, were searched for a match on each patient's name, sex , and birth date. To account for the unequal follow up period (5-7.5 years) we analysed survival up to five years after the clinic visit for everyone.

There was a total of 34 deaths at five year follow up, yielding an average yearly mortality rate of $1.92 \%$. The proportions of deaths by sex and AHI categories are shown in table 3. Although the proportion who died was similar for men and women with an AHI of $<5$, there was a higher proportion of deaths in women with an AHI of $\geqslant 5$. Among women with an

Table 3 Five year mortality for men and women patients according to apnoea/hypopnoea index (AHI) in sleep clinic sample $(n=354)$

\begin{tabular}{llllll}
\hline & \multicolumn{3}{l}{ Men } & & \multicolumn{2}{l}{ Women } \\
\cline { 2 - 3 } \cline { 5 - 6 } AHI & $\%$ dead & $\begin{array}{l}\text { Mean }(S E) \\
\text { age at death } \\
\text { (years) }\end{array}$ & & $\%$ dead & $\begin{array}{l}\text { Mean (SE) } \\
\text { age at death } \\
\text { (years) }\end{array}$ \\
\hline$<5$ & $11(8 / 73)$ & $63.6(0.9)$ & & $10(6 / 59)$ & $66.4(2.2)$ \\
$5-20$ & $2(1 / 49)$ & $71.0(0.0)$ & & $27(3 / 11)$ & $61.0(2.8)$ \\
$>20$ & $8(10 / 141)$ & $71.7(0.7)$ & & $33(7 / 21)$ & $67.1(2.0)$ \\
Total & $7(19 / 263)$ & $72.7(2.0)$ & & $18(16 / 91)$ & $66.7(2.2)$ \\
\hline
\end{tabular}

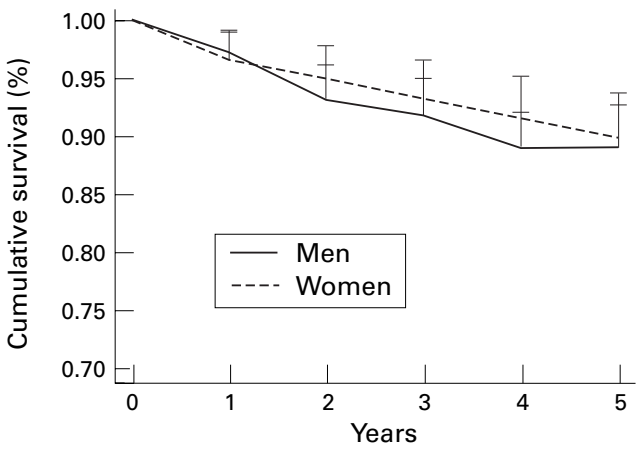

Figure 1 Mean (SE) cumulative five year survival (\%) for sleep clinic patients (73 men, 59 women) with an apnoea/hypopnoea index (AHI) of $<5$.

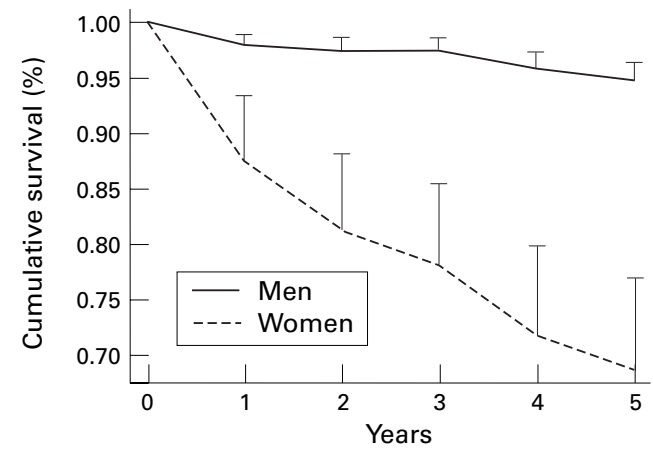

Figure 2 Mean (SE) cumulative five year survival (\%) for sleep clinic patients (190 men, 32 women) with an apnoea/hypopnoea index (AHI) of $\geqslant 5$.

AHI of $>5$, a strikingly high proportion had died during the follow up interval. The mean age at death was significantly lower for women than for men, and this was particularly so in patients with an AHI of $>5$. Thus, age does not account for the difference in mortality; the lower age of the women makes the difference even more notable. The mean (SE) AHI for patients who died in the group with an AHI of $>5$ was 53.3 (24.0) for women and 40.3 (13.9) for men. Cumulative survival over the five year follow up period (SAS software, procedure LIFEREG) for men and women is shown in fig 1 for patients with an AHI of $<5$ and in fig 2 for patients with an $\mathrm{AHI}$ of $\geqslant 5$. Of patients with an AHI of $>5$, women had significantly lower survival rates than men. Compared with the expected mortality based on Wisconsin rates for men and women in the age group 30-65, there was significantly greater mortality for the women, but not the men, with SDB.

The higher death rate for women with SDB is difficult to interpret without further analyses. We are currently investigating this clinic sample on sex differences in diagnosis, comorbidity, treatment, and compliance. From our limited analysis it does not appear that the women as a group definitely had more severe SDB based on AHI. Although the average AHI in the patients who died was higher for women than for men, the difference was not statistically significant. It is possible that a high proportion of the women in this sample were referred as a result of severe comorbidity rather than exclusively for SDB symptoms such as sleepiness. However, if more severe comorbidity alone explained the high death rate for women with 
SDB, the rate for women with an AHI of $<5$ should be similarly high. Alternatively, women with SDB may have had less than optimal treatment or been less compliant with a prescribed treatment than men.

It is possible that more of the men in our study sample had died but were missed when searching the mortality records. Use of the national death index or making contact with the patients or an informant would be necessary to make certain that all deaths outside of Wisconsin were detected. It is, however, unlikely that missed deaths among men with SDB who died out of the State could explain the findings, as 40 more deaths would be needed to match the death rate of the women.

Women with SDB in our sample experienced a much higher mortality rate than did the men. Although the higher mortality rate suggests that health care barriers put women with SDB in our sample at serious risk of dying, our findings are only relevant to care seeking, referral patterns, evaluation, treatment, and other factors that existed in 1987-89. However, further investigation of the factors that are associated with the mortality difference in this study may provide insights into changes needed in the equitable provision of health care for SDB.

1 National Commission on Sleep Disorders Research, Vol 1. Executive Summary and Executive Report. Bethesda, Maryland: National Institutes of Health, 1993.

2 Phillipson E. Sleep apnea: a major public health problem. $N$ Engl f Med 1993;328:1271-3.

3 Bresnitz E. Epidemiology of obstructive sleep apnea. Epidemiol Rev 1994;16:210-27.

4 Young T, Palta M, Dempsey J, et al. The occurrence of Young T, Palta $M$, Dempsey J, et al. The occurrence of
sleep-disordered breathing among middle-aged adults. $N$ Engl f Med 1993;328:1230-5.

5 Strohl K, Redline S. Recognition of obstructive sleep apnea. Am F Respir Crit Care Med 1996;154:279-89.

6 Young T, Hutton R, Finn L, et al. The gender bias in sleep apnea diagnosis: Are women missed because they have different symptoms? Arch Intern Med 1996;156:2445-51.

7 Hoffstein V. Is snoring dangerous to your health? Sleep 1996;19:506-16.

8 Young T, Peppard P, Palta M. et al. Population-based study of sleep-disordered breathing as a risk factor for hypertension. Arch Intern Med 1997;157:1746-52.

9 Young T, Finn L, Hla K, et al. Snoring as part of a dose-response relationship between sleep-disordered dose-response relationship between sleep-disor
breathing and blood pressure. Sleep 1996;19:S202-5.

10 Angell M. Caring for women's health: what is the problem? NEngl f Med 1993;329:271-2. 